\title{
The effect of late onset, short term caloric restriction on the core temperature and physical activity in mice
}

\author{
Andrew Golightly and Richard J. Boys \\ School of Mathematics \& Statistics, Newcastle University, Newcastle Upon Tyne, UK. \\ Kerry M. Cameron and Thomas von Zglinicki \\ Institute for Ageing and Health, Newcastle University, Campus for Ageing and Vitality, Newcas- \\ tle Upon Tyne, UK.
}

Summary. Caloric restriction (CR) has been shown to delay the onset of cancer and other diseases associated with ageing. Currently there are very few studies examining the wholeanimal physiological response to late onset $\mathrm{CR}$. In this paper we study the ways by which mice physiologically compensate for reduced food availability given exposure to late onset $\mathrm{CR}$ and compare these to mice fed ad libitum (AL). The data arise from a 70 day experiment undertaken by the authors. A joint model is developed to describe core body temperature and activity levels and the model parameters assessed for temporal dependence by using dynamic linear models (DLMs). A Bayesian approach is used throughout. The dynamic parameters are shown to be plausibly constant over time and the constant parameter model is then embedded within a random effects structure to explore differences between the responses of $\mathrm{CR}$ and $\mathrm{AL}$ mice. This model is shown to provide a good description of the data.

Key Words: Dynamic linear model, random effects, core body temperature, physical activity, mice.

\section{Introduction}

Caloric restriction $(\mathrm{CR})$, in which adequate nutrition is maintained but total caloric intake is reduced, has been shown to delay the onset of cancer and other diseases associated with ageing. These effects have been demonstrated in the laboratory in organisms such as yeast, worms, flies and mice (Weindruch and Walford, 1988). Although it has been shown that early onset CR has the most dramatic effect on lifespan, there is also evidence to suggest there may be beneficial effects of late onset CR (Weindruch and Walford, 1982; Yu et al., 1985; Spindler, 2005). Recent work in this area has mainly focused on the effect of CR on lifespan (Spindler, 2005) and cancer incidence (Weindruch and Walford, 1982; Volk et al., 1994; Pugh et al., 1999; Spindler, 2005) and there are currently very few studies examining the whole-animal physiological response to late onset CR. The aim of this paper is to study the ways by which rodents physiologically compensate for reduced food availability given exposure to late onset CR. We focus on physical activity and core body temperature and study these factors using data on seven caloric restricted mice and seven mice fed ad libitum (AL). A reduction in core body temperature has been shown to be a key factor in the lifeprolonging effects of $\mathrm{CR}$, a result which has been widely reproduced, although can vary with strain (Liao et al., 2010). We aim to determine if body temperature is also reduced in mice undergoing short term CR. Under CR, mice may compensate for the reduced energy intake by reducing energy expenditure; a major component of which is physical activity. 
Previous analyses have largely studied physical activity and core temperature separately. For example, Dixon et al. (2003) examine daily running wheel activity in rodents using repeated measures ANOVA. Conti et al. (2006) also used repeated measures ANOVA to analyse core body temperature and motor activity data (taken over 10 minute intervals) in transgenic mice. Mousel et al. (2000) analysed core temperature and locomotor activity in lines of mice selected for high or low heat loss. They used daily means and a random effects approach. In addition, they studied circadian rhythms by using 30-minute observations and including a time effect decomposed into a Fourier series spectra with components taking the form of a sinusoid.

Greater insight into the effect of caloric restriction may be obtained by studying these physiological responses jointly and building in the possibly complex dependencies between these responses over time. In this paper we propose a (simple) joint model for hourly average core temperature and (transformed) physical activity counts and study whether there are any additional temporal effects via dynamic linear models (DLMs). Finally, differences between CR and AL mice are explored by using a Bayesian random effects model (Gelman et al., 2003). We also assess model fit by integrating over parameter uncertainty and looking at predictive distributions. We fit these models using activity and temperature data that are simultaneously collected and unbiased by cage type or presence/absence of a running wheel.

The remainder of the paper is organised as follows. In Section 2 we briefly describe the experiment and the resulting data. Details of the proposed model and an assessment of temporal dependence of the parameters can be found in Section 3. A random effects extension to the model is studied in Section 4 before reporting conclusions in Section 5.

\section{The experimental data}

Mice were taken from a long-established colony of the C57/BL (ICRFa) strain and placed into one of 14 cages, with between 4 and 6 mice in each cage. Mice were fed ad libitum (AL) until they were fourteen months old and cages were then divided into two groups (matched for body mass, food intake and age) with 7 cages in each group. One group was allocated to AL feeding and the other to caloric restricted (CR) for 70 days. CR mice were offered a $40 \%$ food restriction relative to the AL group, delivered as one daily ration at around 9.30am. One randomly chosen mouse in each cage was implanted with a wireless E-mitter which continuously monitored body temperature and physical activity. The intention was to measure mean body temperature and physical activity counts every minute. However, for a variety of reasons, the data do contain missing values. Therefore, in this paper, we analyse values of body temperature and physical activity counts averaged over each consecutive hour. The main reason for data to be missing was when mice were removed from the cages for weighing. There was also around 20 hours worth of data lost due to a power cut towards the end of the experiment. Data were also excluded from the first two weeks and the last week of the experiment as mice were very heavily disturbed, initially due to many measurements being taken and finally as the remaining mice in each cage were removed and then dissected. Hence the data consist of some 1156 hourly averages on body temperature and physical activity on 14 mice ( $7 \mathrm{AL}$ and $7 \mathrm{CR}$ ).

Exploratory plots of the data are useful in building appropriate statistical models. Figure 1 shows the first 96 data points (representing 4 days of data) for a single typical mouse in each of the AL and CR groups. It shows a clear linear relationship between hourly average 
(a)
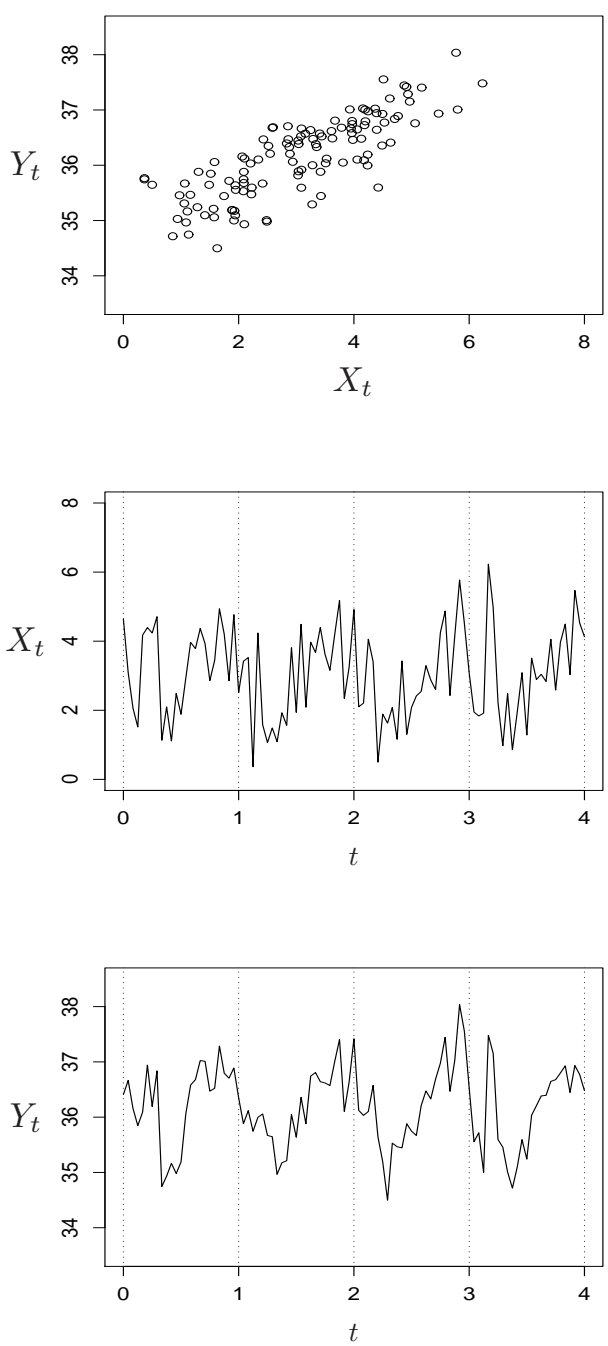

(b)
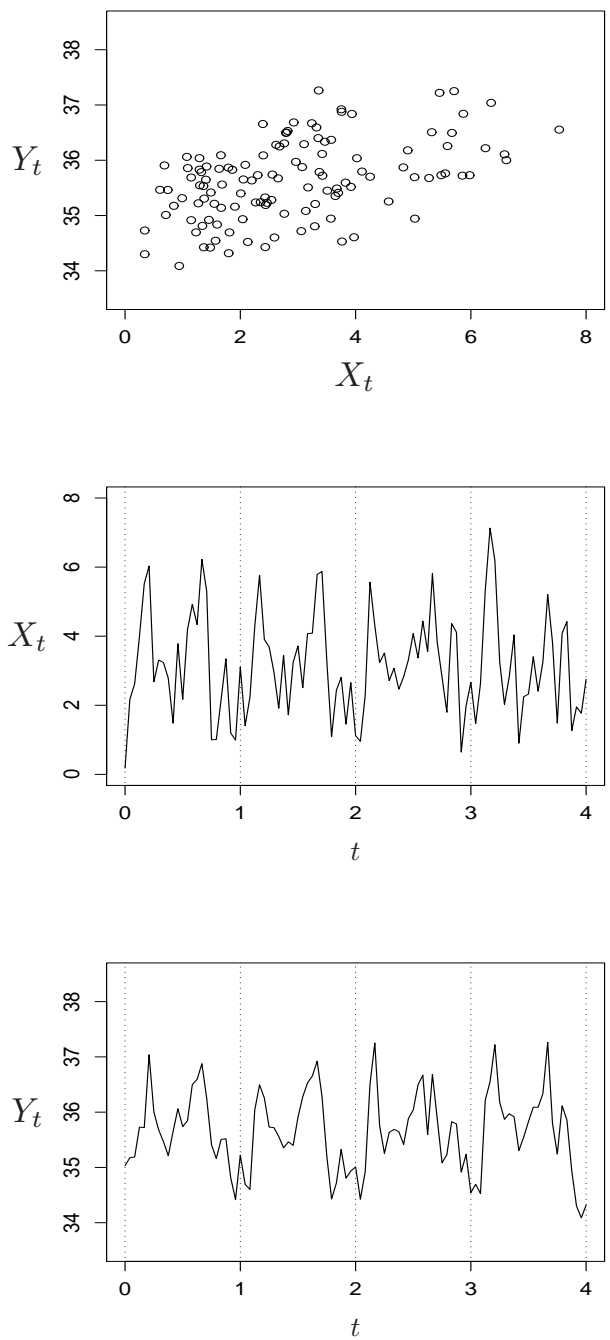

Fig. 1. Top panel: hourly average temperature $\left(Y_{t}\right)$ against square rooted hourly average activity $\left(X_{t}\right)$. Middle panel: square rooted hourly average activity against time (in days). Bottom panel: hourly average temperature $\left(Y_{t}\right)$ against time (in days). All plots show the first 4 days of data (starting at 7am), having discarded the first two weeks, for (a) mouse 1 (AL group) and (b) mouse 1 (CR group). The plots include data collected during the disruptive period (9am-12 noon) during which increased activity and temperature levels are clearly visible. 
temperature $\left(Y_{t}\right)$ against hourly average activity on a square root scale $\left(X_{t}\right)$. It also shows that (square rooted) activity and temperature exhibit a sinusoidal pattern that repeats around every 24 hours, with mice inactive during the day and active in the night. A complication in the data collection was caused by a period of daily disturbance due to feeding, weighing and cleaning out of cages around 9am-12 noon. Both activity and temperature levels are higher during these periods than at any other time. We will call this period the disruptive period and return to its impact for fitting the model in the next section.

\section{Assessing temporal variation}

\subsection{Model}

Before assessing any differences between AL and CR mice (see section 4), we first develop a joint model for hourly average core temperature and transformed (via the square root) physical activity counts. We specify the model through a marginal model for activity and then a conditional model for temperature given activity level. Conditioning temperature on (transformed) activity rather than vice versa is largely motivated by the homeothermic nature of mice: although body temperature is controlled within narrow limits, increasing physical activity will increase temperature within these regulatory limits. As remarked previously, the data show a clear circadian rhythm in both the temperature and the activity measurements, which suggest marginal models with a sinusoidal form with a 24 hour period. Also the relationship between core temperature and (transformed) physical activity has a clear linear form. In order to develop simple models to describe between group variability, it would be useful to be able to assume that the parameters in these models are constant over time. Therefore, we examine the temporal consistency of these parameters by fitting dynamic linear models (DLMs) in which the key parameters of the sinusoidal model, amplitude and phase shift, and in the conditional linear model are allowed to vary smoothly over time. A complicating factor within the data is that the mice are artificially stimulated during the daily $9 \mathrm{am}-12$ noon disruptive period. We therefore choose to ignore data during these periods in this preliminary analysis.

For simplicity we consider first a joint model for hourly average temperature and square rooted activity for a single mouse. Let $Y_{t_{i}}$ denote hourly average temperature taken over the interval $\left(t_{i}, t_{i}+1\right.$ ] (with $t_{i}$ in hours, $\left.i=1, \ldots, n\right)$ and $X_{t_{i}}$ denote the corresponding square rooted activity value. Without loss of generality, we scale time so that $t_{1}=0$. Due to the linear relationship between temperature and square rooted activity, and the sinusoidal pattern (with a period of 24 hours) in the activity data, we propose DLMs for square rooted activity and for temperature given (transformed) activity with observation equations, for $i=1,2, \ldots, n$

$$
\begin{aligned}
Y_{t_{i}} & =\beta_{1}+\beta_{t_{i}, 1}+\left(\beta_{2}+\beta_{t_{i}, 2}\right) X_{t_{i}}+\sigma_{y} \epsilon_{i}^{y} \\
X_{t_{i}} & =\mu_{x}+\left(\theta_{1}+\theta_{t_{i}, 1}\right) \sin \left(\frac{\pi t_{i}}{12}\right)+\left(\theta_{2}+\theta_{t_{i}, 2}\right) \cos \left(\frac{\pi t_{i}}{12}\right)+\sigma_{x} \epsilon_{i}^{x}
\end{aligned}
$$

where the independent error terms $\epsilon_{i}^{x}$ and $\epsilon_{i}^{y}$ follow standard normal distributions. Note that the marginal model for (square rooted) activity can also be written in sinusoidal form, with

$$
X_{t_{i}}=\mu_{x}+A_{t_{i}} \cos \left(\frac{\pi t_{i}}{12}-B_{t_{i}}\right)+\sigma_{x} \epsilon_{i}^{x}
$$


where the parameters in (1) and (2) are related using

$$
A_{t_{i}}=\sqrt{\left(\theta_{1}+\theta_{t_{i}, 1}\right)^{2}+\left(\theta_{2}+\theta_{t_{i}, 2}\right)^{2}}, \quad B_{t_{i}}=\tan ^{-1}\left(\frac{\theta_{1}+\theta_{t_{i}, 1}}{\theta_{2}+\theta_{t_{i}, 2}}\right) .
$$

The parameters in the model (for each mouse) have the following interpretations: $\mu_{x}$ describes overall mean (square rooted) activity level, and at time $t_{i}, A_{t_{i}}$ is the amplitude of the sinusoid about mean activity, $B_{t_{i}}$ is the phase shift, $\beta_{1}+\beta_{t_{1}, 1}$ gives the basal temperature level and $\beta_{2}+\beta_{t_{i}, 2}$ is the coefficient describing the linear relationship between temperature and square rooted activity. Note that this joint model induces a marginal model for hourly average temperature with the sinusoidal pattern observed in the data. A related approach is described by Challet et al. (1997) who use a sinusoid with constant amplitude and phase shift to model body temperature in rats.

Clearly, the joint model (1) is over-parameterised and so we add constraints on the timevarying parameters, namely, that they vary slowly and smoothly over time. We impose some smoothness on the parameters by taking the standard random walk model for the state equations in the DLMs (adjusted for non-regular sampling), namely

$$
\begin{aligned}
\boldsymbol{\beta}_{t_{i}} & =\boldsymbol{\beta}_{t_{i-1}}+k_{i} \operatorname{diag}\left(\boldsymbol{\sigma}_{\beta}\right) \boldsymbol{\omega}_{i}^{y} \\
\boldsymbol{\theta}_{t_{i}} & =\boldsymbol{\theta}_{t_{i-1}}+k_{i} \operatorname{diag}\left(\boldsymbol{\sigma}_{\theta}\right) \boldsymbol{\omega}_{i}^{x}
\end{aligned}
$$

where $\boldsymbol{\beta}_{t_{i}}=\left(\beta_{t_{i}, 1}, \beta_{t_{i}, 2}\right)^{\prime}, \boldsymbol{\theta}_{t_{i}}=\left(\theta_{t_{i}, 1}, \theta_{t_{i}, 2}\right)^{\prime}, \boldsymbol{\sigma}_{\beta}=\left(\sigma_{\beta, 1}, \sigma_{\beta, 2}\right)^{\prime}, \boldsymbol{\sigma}_{\theta}=\left(\sigma_{\theta, 1}, \sigma_{\theta, 2}\right)^{\prime}$, the noise terms $\boldsymbol{\omega}_{i}^{x}$ and $\boldsymbol{\omega}_{i}^{y}$ have components with independent standard normal distributions, and $k_{i}^{2}=t_{i}-t_{i-1}$. To allow identifiability of all model parameters, we set $\beta_{t_{1}, j}=\theta_{t_{1}, j}=0$ for $j=1,2$. Thus differences between $\beta_{t_{i}}$ values and between $\theta_{t_{i}}$ values are scaled by how far apart they are in time and on the amount of smoothness in these latent processes, regulated by the size of $\boldsymbol{\sigma}_{\beta}$ and $\boldsymbol{\sigma}_{\theta}$. Also note that, although (1) and (4) define DLMs for $X_{t}$ and for $Y_{t}$ given $X_{t}$, the induced model for the bivariate time series $\left(X_{t}, Y_{t}\right)^{\prime}$ is not a DLM as the covariance matrix in the induced observation equation depends on the dynamic parameters.

Our aim in this preliminary analysis is to assess temporal dependence amongst the $\boldsymbol{\beta}_{t_{i}}$ and $\boldsymbol{\theta}_{t_{i}}$ by testing $\boldsymbol{\sigma}_{\beta}=\boldsymbol{\sigma}_{\theta}=\mathbf{0}$. A Bayesian approach to this problem typically involves either computation of the marginal likelihood for each model (Kass and Raftery, 1995; Frühwirth-Schnatter, 1995) or a variable selection method (George and McCulloch, 1993, 1997; Frühwirth-Schnatter and Tüchler, 2008; Frühwirth-Schnatter and Wagner, 2010). We follow the latter paper by working with their non-centered form of the model (which removes the parameters to be restricted $\left(\boldsymbol{\sigma}_{\beta}, \boldsymbol{\sigma}_{\theta}\right)$ from the system equations) and include a binary parameter $\gamma \in\{0,1\}$. This binary parameter plays a variable selection role in which $\gamma=$ 1 corresponds to the full model which allows for temporal dependence whereas $\gamma=0$ corresponds to the restricted "no temporal dependence" model with $\boldsymbol{\sigma}_{\beta}=\boldsymbol{\sigma}_{\theta}=\mathbf{0}$. The transformed model has observation equations

$$
\begin{aligned}
& Y_{t_{i}}=\beta_{1}+\gamma \tilde{\sigma}_{\beta, 1} \tilde{\beta}_{t_{i}, 1}+\left(\beta_{2}+\gamma \tilde{\sigma}_{\beta, 2} \tilde{\beta}_{t_{i}, 2}\right)\left(X_{t_{i}}-\bar{x}\right)+\sigma_{y} \epsilon_{i}^{y} \\
& X_{t_{i}}=\mu_{x}+\left(\theta_{1}+\gamma \tilde{\sigma}_{\theta, 1} \tilde{\theta}_{t_{i}, 1}\right) \sin \left(\frac{\pi t_{i}}{12}\right)+\left(\theta_{2}+\gamma \tilde{\sigma}_{\theta, 2} \tilde{\theta}_{t_{i}, 2}\right) \cos \left(\frac{\pi t_{i}}{12}\right)+\sigma_{x} \epsilon_{i}^{x},
\end{aligned}
$$

where $\bar{x}$ is the sample mean square rooted activity level, and system equations

$$
\begin{aligned}
\tilde{\boldsymbol{\beta}}_{t_{i}} & =\tilde{\boldsymbol{\beta}}_{t_{i-1}}+k_{i} \tilde{\boldsymbol{\omega}}_{i}^{y} \\
\tilde{\boldsymbol{\theta}}_{t_{i}} & =\tilde{\boldsymbol{\theta}}_{t_{i-1}}+k_{i} \tilde{\boldsymbol{\omega}}_{i}^{x},
\end{aligned}
$$


with $\tilde{\beta}_{t_{1}, j}=\tilde{\theta}_{t_{1}, j}=0$ for $j=1,2$. Here $\tilde{\boldsymbol{\beta}}_{t_{i}}=\left(\tilde{\beta}_{t_{i}, 1}, \tilde{\beta}_{t_{i}, 2}\right)^{\prime}, \tilde{\boldsymbol{\theta}}_{t_{i}}=\left(\tilde{\theta}_{t_{i}, 1}, \tilde{\theta}_{t_{i}, 2}\right)^{\prime}$, and $\tilde{\boldsymbol{\omega}}_{i}^{x}$ and $\tilde{\boldsymbol{\omega}}_{i}^{y}$ have components with independent standard normal distributions. Setting $\beta_{t_{i}, j}=\tilde{\sigma}_{\beta, j} \tilde{\beta}_{t_{i}, j}, \theta_{t_{i}, j}=\tilde{\sigma}_{\theta, j} \tilde{\theta}_{t_{i}, j}, \sigma_{\beta, j}=\left|\tilde{\sigma}_{\beta, j}\right|$ and $\sigma_{\theta, j}=\left|\tilde{\sigma}_{\theta, j}\right|$ for $j=1,2$ recovers the centered model. Note that the purpose of centering the regressor $X_{t_{i}}$ is to reduce posterior dependence between the many intercept $\left(\beta_{1}, \tilde{\beta}_{t_{i}, 1}\right)$ and slope $\left(\beta_{2}, \tilde{\beta}_{t_{i}, 2}\right)$ parameters in order to obtain a better mixing chain.

A potential drawback of using the non-centered parameterisation is that it introduces an identifiability problem: multiplying any of the components of $\tilde{\boldsymbol{\sigma}}_{\beta}$ and $\tilde{\boldsymbol{\sigma}}_{\theta}$ and their corresponding components in $\tilde{\boldsymbol{\beta}}_{t_{i}}$ and $\tilde{\boldsymbol{\theta}}_{t_{i}}$ by -1 will not change the distributions of $Y_{t_{i}}$ and $X_{t_{i}}$. Consequently, the marginal (or integrated) likelihood of $\tilde{\boldsymbol{\sigma}}_{\beta}$ and $\tilde{\boldsymbol{\sigma}}_{\theta}$ will be multimodal and symmetric about the origin along its component axes, though, of course, it only has a single mode when $\tilde{\boldsymbol{\sigma}}_{\beta}=\tilde{\boldsymbol{\sigma}}_{\theta}=\mathbf{0}$. However, Frühwirth-Schnatter and Wagner (2010) point out that this identifiability problem has no bearing on inferences for the centered model apart from a possible benefit of inducing a more rapidly mixing chain.

\subsection{Inference}

Let $\boldsymbol{y}=\left(y_{t_{1}}, y_{t_{2}}, \ldots, y_{t_{n}}\right)^{\prime}$ denote the observed hourly average temperature trace and $\boldsymbol{x}=$ $\left(x_{t_{1}}, x_{t_{2}}, \ldots, x_{t_{n}}\right)^{\prime}$ denote the corresponding square rooted activity trace. Denote the fixed parameters in the model by $\boldsymbol{\psi}=\left(\boldsymbol{\psi}_{y}, \boldsymbol{\psi}_{x}\right)^{\prime}$ where $\boldsymbol{\psi}_{y}=\left(\beta_{1}, \beta_{2}, \sigma_{y}, \tilde{\sigma}_{\beta, 1}, \tilde{\sigma}_{\beta, 2}\right)^{\prime}$ and $\boldsymbol{\psi}_{x}=$ $\left(\mu_{x}, \theta_{1}, \theta_{2}, \sigma_{x}, \tilde{\sigma}_{\theta, 1}, \tilde{\sigma}_{\theta, 2}\right)^{\prime}$, and the time-varying parameters by $\tilde{\boldsymbol{\beta}}=\left(\tilde{\boldsymbol{\beta}}_{t_{2}}^{\prime}, \ldots, \tilde{\boldsymbol{\beta}}_{t_{n}}^{\prime}\right)^{\prime}$ and $\tilde{\boldsymbol{\theta}}=$ $\left(\tilde{\boldsymbol{\theta}}_{t_{2}}^{\prime}, \ldots, \tilde{\boldsymbol{\theta}}_{t_{n}}^{\prime}\right)^{\prime}$. Note that $\tilde{\beta}_{t_{1}, j}=\tilde{\theta}_{t_{1}, j}=0$ for $j=1,2$. We assume a prior specification of the form

$$
\pi(\gamma, \boldsymbol{\psi}, \tilde{\boldsymbol{\beta}}, \tilde{\boldsymbol{\theta}})=\pi(\gamma) \pi\left(\boldsymbol{\psi}_{y}\right) \pi\left(\boldsymbol{\psi}_{x}\right) \pi(\tilde{\boldsymbol{\beta}}) \pi(\tilde{\boldsymbol{\theta}})
$$

where the contributions of the latent structures are

$$
\pi(\tilde{\boldsymbol{\beta}})=\prod_{i=2}^{n} \phi\left(\tilde{\beta}_{t_{i}, 1} \mid \tilde{\beta}_{t_{i-1}, 1}, k_{i}^{2}\right) \phi\left(\tilde{\beta}_{t_{i}, 2} \mid \tilde{\beta}_{t_{i-1}, 2}, k_{i}^{2}\right)
$$

and

$$
\pi(\tilde{\boldsymbol{\theta}})=\prod_{i=2}^{n} \phi\left(\tilde{\theta}_{t_{i}, 1} \mid \tilde{\theta}_{t_{i-1}, 1}, k_{i}^{2}\right) \phi\left(\tilde{\theta}_{t_{i}, 2} \mid \tilde{\theta}_{t_{i-1}, 2}, k_{i}^{2}\right)
$$

where $\phi\left(\cdot \mid \mu, \sigma^{2}\right)$ denotes the normal density with mean $\mu$ and variance $\sigma^{2}$. Finally, we adopt the following semi-conjugate independent prior specification for the remaining unknowns

$$
\begin{aligned}
\gamma & \sim \operatorname{Binomial}(1,0.5), & \mu_{x} \mid \sigma_{x} & \sim \mathrm{N}\left(b_{x}, d_{x}^{2} \sigma_{x}^{2}\right), \\
\beta_{j} \mid \sigma_{y} & \sim \mathrm{N}\left(b_{\beta_{j}}, d_{\beta_{j}}^{2} \sigma_{y}^{2}\right), \quad j=1,2, & \theta_{j} \mid \sigma_{x} & \sim \mathrm{N}\left(b_{\theta_{j}}, d_{\theta_{j}}^{2} \sigma_{x}^{2}\right), \quad j=1,2, \\
\tilde{\sigma}_{\beta, j} \mid \sigma_{y} & \sim \mathrm{N}\left(0, d_{\tilde{\sigma}_{\beta}}^{2} \sigma_{y}^{2}\right), \quad j=1,2, & \tilde{\sigma}_{\theta, j} \mid \sigma_{x} & \sim \mathrm{N}\left(0, d_{\tilde{\sigma}_{\theta}}^{2} \sigma_{x}^{2}\right), \quad j=1,2 \\
\sigma_{y}^{-2} & \sim \Gamma\left(g_{y}, h_{y}\right), & \sigma_{x}^{-2} & \sim \Gamma\left(g_{x}, h_{x}\right),
\end{aligned}
$$

where $\Gamma(g, h)$ denotes the Gamma distribution with mean $g / h$. We elicited values for the prior distribution parameters in discussion with our experimental colleagues (KMC and TvZ). We had fairly strong prior beliefs about mean activity and basal temperature. For example, it was anticipated that most temperature values would lie between $34^{\circ} \mathrm{C}$ and $38^{\circ} \mathrm{C}$. 
We represent these beliefs by taking $b_{x}=3, b_{\beta_{1}}=36$ and $d_{x}=d_{\beta_{1}}=1$. Weak prior beliefs about $\theta_{1}, \theta_{2}$ and $\beta_{2}$ are represented via $b_{\theta_{1}}=b_{\theta_{2}}=b_{\beta_{2}}=0$ and $d_{\theta_{1}}=d_{\theta_{2}}=d_{\beta_{2}}=10$. To represent weak prior beliefs about $\sigma_{x}$ and $\sigma_{y}$ we take $g_{x}=g_{y}=1$ and $h_{x}=h_{y}=0.05$. Our strong opinion that the components of $\tilde{\boldsymbol{\sigma}}_{\theta}$ and $\tilde{\boldsymbol{\sigma}}_{\beta}$, which govern the smoothness of the $\boldsymbol{\theta}_{t_{i}}$ and $\boldsymbol{\beta}_{t_{i}}$, should be small is represented via $d_{\tilde{\sigma}_{\beta}}=d_{\tilde{\sigma}_{\theta}}=1$. We follow Frühwirth-Schnatter and Wagner (2010) by specifying zero mean Normal priors for the $\tilde{\sigma}_{\beta, j}$ and $\tilde{\sigma}_{\theta, j}$ and is equivalent to placing half-Normal priors on the components of $\boldsymbol{\sigma}_{\beta}$ and $\boldsymbol{\sigma}_{\theta}$. The motivation for this approach is two-fold: this choice of prior leads to a Gibbs sampler that can easily be implemented and also is less influential on posterior inferences if the true state variances are close to zero than when using inverse Gamma priors (Gelman, 2006).

The joint posterior distribution of all model parameters is not analytically tractable. However, our prior specification produces tractable full conditionals for the components of $\gamma, \boldsymbol{\psi}_{y}, \boldsymbol{\psi}_{x}$ and the dynamic parameters $\tilde{\boldsymbol{\beta}}$ and $\tilde{\boldsymbol{\theta}}$. Hence, it is possible to obtain realisations from this posterior distribution via a Gibbs sampler in conjunction with a forwards backwards algorithm (Carter and Kohn, 1994; Frühwirth-Schnatter, 1994; Shephard, 1994; West and Harrison, 1997) for sampling from the full conditionals of the dynamic parameters. Details of the MCMC sampler can be found in Appendix A.

\subsection{Results}

We now fit the non-centered model described in Section 3.1 to the data on each mouse separately using the Gibbs sampler described in Appendix A. In order to promote mixing over the model selection parameter $\gamma$, we follow the recommendation of Frühwirth-Schnatter and Wagner (2010) and first run the chain for an initial period with $\gamma=1$ and then remove this restriction. Convergence was assessed using a variety of formal and informal methods on omnibus model descriptors such as the log-likelihood function. We found that running the sampler with $1 \mathrm{~K}$ iterations for burn-in and then a further 50K iterations (without thinning) gave converged output with sufficiently low autocorrelations. Table 1 gives the posterior means and standard deviations for the variance parameters for each mouse, obtained from the output of the Gibbs sampler applied to the full model allowing temporal dependence, that is, conditional on $\gamma=1$. The posterior means and standard deviations for $\sigma_{y}$ and $\sigma_{x}$ in the table suggest that there is more variation (about the sinusoidal pattern) in both the activity and temperature levels for mice in the CR group compared to the AL group.

Figure 2 shows the posterior means and the $95 \%$ credible regions for the basal temperature $\beta_{1}+\beta_{1, t}-\left(\beta_{2}+\beta_{2, t}\right) \bar{x}$ and slope coefficient $\beta_{2}+\beta_{2, t}$ and the parameter combinations $\theta_{1}+\theta_{1, t}$ and $\theta_{2}+\theta_{2, t}$ for mouse 1 in the AL group and mouse 1 in the CR group. These plots were obtained using the MCMC output with $\gamma=1$ described above and can be used to give an informal assessment of the hypothesis that the dynamic parameters are plausibly constant. The figure suggests that this is indeed the case for these two mice. Plots for the other mice give a similar conclusion. This hypothesis can be judged more formally by using an empirical estimate for the posterior probability that the binary variable selection parameter is zero, that is, $\widehat{\operatorname{Pr}}(\gamma=0 \mid \boldsymbol{x}, \boldsymbol{y})$; see Table 2. These probabilities give strong support for the hypothesis that the dynamic parameters are constant. This is also suggestive that the circadian rhythms we see in the data did not change significantly over the course of the experiment. We also examined the sensitivity of these conclusions to moderate changes in the concentration of the prior distributions for $\boldsymbol{\sigma}_{\beta}$ and $\boldsymbol{\sigma}_{\theta}$ about their zero means. Despite considering ten-fold increases in their standard deviations (by varying $d_{\tilde{\sigma}_{\beta}}$ and $\left.d_{\tilde{\sigma}_{\theta}}\right)$, we found roughly similar values for $\widehat{\operatorname{Pr}}(\gamma=0 \mid \boldsymbol{x}, \boldsymbol{y})$ and therefore determined that 
Table 1. Marginal posterior means (and standard deviations) for the variance parameters $\sigma_{y}, \sigma_{\beta, 1}, \sigma_{\beta, 2}, \sigma_{x}, \sigma_{\theta, 1}$ and $\sigma_{\theta, 2}$ in the full model $(\gamma=1)$.

\begin{tabular}{|ccccc|}
\hline Group & Mouse & $\sigma_{y}$ & $\sigma_{\beta, 1}$ & $\sigma_{\beta, 2}$ \\
\hline AL & 1 & $0.204(0.010)$ & $0.222(0.010)$ & $0.0018(0.0010)$ \\
& 2 & $0.231(0.014)$ & $0.190(0.014)$ & $0.0023(0.0017)$ \\
& 3 & $0.235(0.010)$ & $0.159(0.012)$ & $0.0014(0.0024)$ \\
& 4 & $0.281(0.013)$ & $0.197(0.017)$ & $0.0011(0.0012)$ \\
& 5 & $0.257(0.022)$ & $0.154(0.030)$ & $0.0011(0.0009)$ \\
& 6 & $0.159(0.009)$ & $0.217(0.009)$ & $0.0013(0.0015)$ \\
CR & 7 & $0.214(0.010)$ & $0.200(0.010)$ & $0.0012(0.0011)$ \\
& 1 & $0.321(0.013)$ & $0.264(0.017)$ & $0.0026(0.0010)$ \\
& 2 & $0.241(0.016)$ & $0.270(0.018)$ & $0.0015(0.0012)$ \\
& 3 & $0.298(0.019)$ & $0.262(0.023)$ & $0.0029(0.0018)$ \\
& 4 & $0.191(0.012)$ & $0.315(0.013)$ & $0.0019(0.0009)$ \\
& 5 & $0.339(0.021)$ & $0.267(0.026)$ & $0.0021(0.0007)$ \\
Group & Mouse & $0.271(0.024)$ & $0.316(0.025)$ & $0.0012(0.0011)$ \\
\hline AL & 1 & $0.975(0.022)$ & $0.0087(0.0065)$ & $0.0113(0.0051)$ \\
& 2 & $1.039(0.024)$ & $0.0049(0.0044)$ & $0.0062(0.0052)$ \\
& 3 & $1.031(0.022)$ & $0.0084(0.0057)$ & $0.0059(0.0052)$ \\
& 4 & $1.049(0.023)$ & $0.0055(0.0055)$ & $0.0049(0.0044)$ \\
& 5 & $1.062(0.024)$ & $0.0051(0.0043)$ & $0.0113(0.0063)$ \\
& 6 & $0.933(0.021)$ & $0.0068(0.0040)$ & $0.0055(0.0053)$ \\
& 7 & $0.998(0.022)$ & $0.0064(0.0044)$ & $0.0049(0.0046)$ \\
\hline CR & 1 & $1.485(0.032)$ & $0.0083(0.0076)$ & $0.0072(0.0086)$ \\
& 2 & $1.223(0.028)$ & $0.0066(0.0056)$ & $0.0167(0.0087)$ \\
& 3 & $1.412(0.032)$ & $0.0068(0.0053)$ & $0.0147(0.0077)$ \\
& 4 & $1.281(0.028)$ & $0.0068(0.0062)$ & $0.0215(0.0077)$ \\
& 5 & $2.481(0.055)$ & $0.0116(0.0096)$ & $0.0253(0.0118)$ \\
& 6 & $1.737(0.039)$ & $0.0075(0.0063)$ & $0.0116(0.0086)$ \\
& 7 & $1.952(0.042)$ & $0.0215(0.0112)$ & $0.0200(0.0104)$ \\
\hline
\end{tabular}


(A) Mouse 1 (AL group)
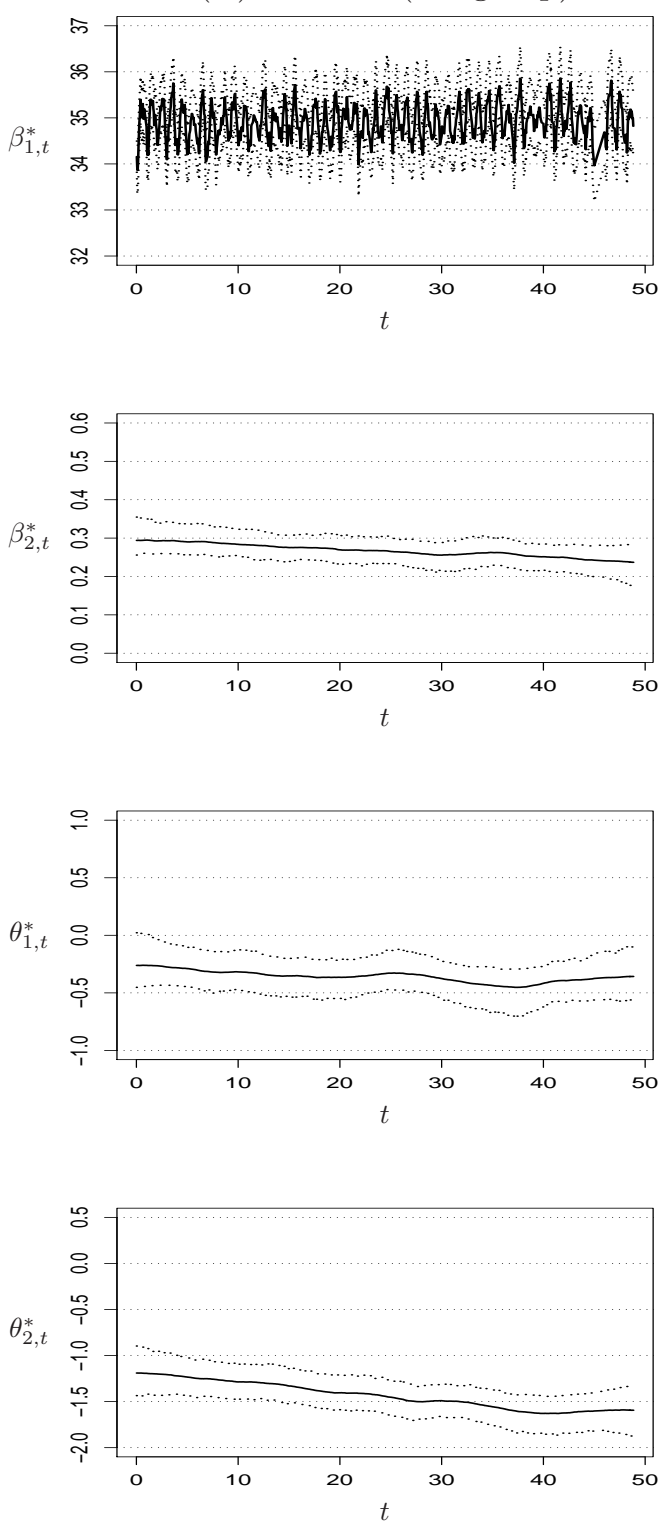

(B) Mouse 1 (CR group)
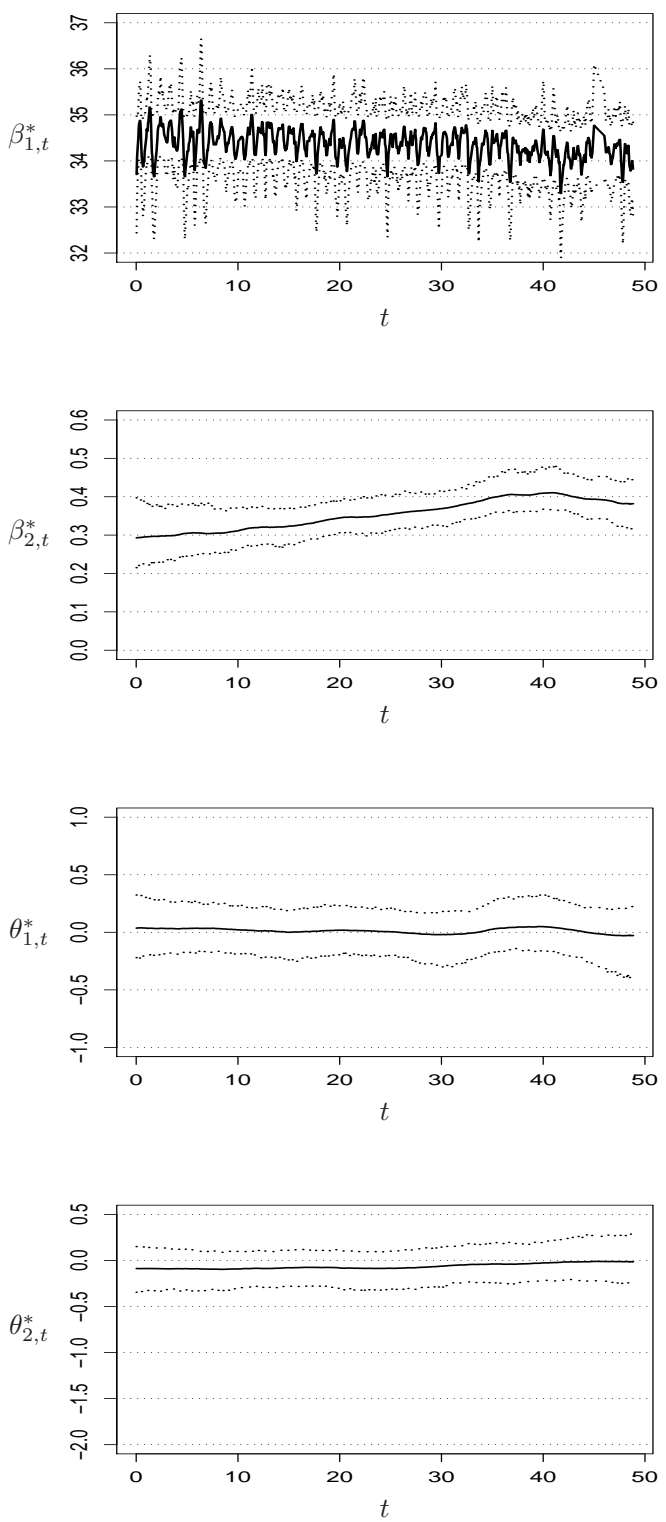

Fig. 2. Posterior means (black lines) and $95 \%$ credible regions (dotted lines) against time (in days) for the dynamic parameters $\beta_{1, t}^{*}=\beta_{1}+\beta_{1, t}-\left(\beta_{2}+\beta_{2, t}\right) \bar{x}, \beta_{2, t}^{*}=\beta_{2}+\beta_{2, t}, \theta_{1, t}^{*}=\theta_{1}+\theta_{1, t}$ and $\theta_{2, t}^{*}=\theta_{2}+\theta_{2, t}$ for (a) mouse 1 (AL group) and (b) mouse 1 (CR group). 
Table 2. Values of the posterior probability of no temporal dependence for each mouse.

\begin{tabular}{|ccc|ccc|}
\hline Group & Mouse & $\widehat{\operatorname{Pr}}(\gamma=0 \mid \boldsymbol{x}, \boldsymbol{y})$ & Group & Mouse & $\widehat{\operatorname{Pr}}(\gamma=0 \mid \boldsymbol{x}, \boldsymbol{y})$ \\
\hline AL & 1 & 1.000 & CR & 1 & 1.000 \\
& 2 & 0.999 & & 2 & 0.802 \\
& 3 & 1.000 & & 3 & 1.000 \\
4 & 0.999 & & 4 & 0.998 \\
5 & 1.000 & & 5 & 1.000 \\
& 6 & 1.000 & & 6 & 1.000 \\
& 7 & 1.000 & & 7 & 0.724 \\
\hline
\end{tabular}

our conclusions were reasonably robust.

In summary, we concluded that it was reasonable to assume that the amplitude, phase shift, basal temperature levels and the slope terms that characterise the relationship between temperature and transformed activity were constant over time. In the next section we examine differences in these (constant) parameters between the AL and CR groups more formally through a random effects model.

\section{A random effects model}

\subsection{Model}

In this section we turn our attention to exploring and quantifying differences between the $\mathrm{AL}$ and CR treatment groups in this pilot study using a random effects structure. We also explicitly model the level of disruption in the disruptive period. For ease of notation, we will denote the AL and CR groups as treatment groups 1 and 2 respectively.

Let $Y_{t_{i}}^{j k}$ be the hourly average temperature at time $t_{i}$ for mouse $j$ in treatment group $k$ and let $X_{t_{i}}^{j k}$ be the corresponding activity level. We now extend the previous model by allowing each mouse to have different (constant) parameters but with these parameters being drawn from appropriate random effect distributions. Thus the model for the observable quantities is

$$
\begin{aligned}
& Y_{t_{i}}^{j k}=\beta_{1}^{j k}+\beta_{2}^{j k} X_{t_{i}}^{j k}+\sigma_{y}^{k} \epsilon_{i j k}^{y}, \\
& X_{t_{i}}^{j k}=\mu_{x}^{j k}+A^{j k} \cos \left(\frac{\pi t_{i}}{12}-B^{j k}\right)+C\left(t_{i}\right)+\sigma_{x}^{k} \epsilon_{i j k}^{x},
\end{aligned}
$$

for $i=1,2, \ldots, n, j=1,2, \ldots, 7$ and $k=1,2$, where the independent errors $\epsilon_{i j k}^{x}$ and $\epsilon_{i j k}^{y}$ follow standard normal distributions. The function $C(t)$ is an attempt to model the disruption in the disruptive period $(9 \mathrm{am}-1 \mathrm{pm})$ explicitly. To gain an idea of what form this function should take we examined residuals within the disruptive periods calculated using parameter estimates obtained from data with the disruptive periods excluded. These residuals suggest that a quadratic function function would be suitable and so we take

$$
C(t)=\left\{\delta_{1}^{j k} d(t)^{2}+\delta_{2}^{j k} d(t)+\delta_{3}^{j k}\right\} I(t),
$$

where $d(t)$ is the time $t$ in hours on a 24 hour clock and $I(t)$ is the indicator function taking the value 1 if $t$ lies in the disruptive period $(9<d(t)<13)$ and 0 otherwise. As before, to facilitate the construction of the MCMC scheme, we rewrite the model for mean activity in 
DLM form as

$$
X_{t_{i}}^{j k}=\mu_{x}^{j k}+\theta_{1}^{j k} \sin \left(\frac{\pi t_{i}}{12}\right)+\theta_{2}^{j k} \cos \left(\frac{\pi t_{i}}{12}\right)+C\left(t_{i}\right)+\sigma_{x}^{k} \epsilon_{i j k}^{x} .
$$

Finally, the model is completed by adding the random effects structure: for $j=1,2, \ldots, 7$ and $k=1,2$

$$
\begin{array}{ll}
\mu_{x}^{j k} \sim \mathrm{N}\left(\mu^{k},\left(\sigma_{\mu}^{k}\right)^{2}\right), & \theta_{\ell}^{j k} \sim \mathrm{N}\left(\theta_{\ell}^{k},\left(\sigma_{\theta_{\ell}}^{k}\right)^{2}\right), \ell=1,2, \\
\delta_{\ell}^{j k} \sim \mathrm{N}\left(\delta_{\ell}^{k},\left(\sigma_{\delta_{\ell}}^{k}\right)^{2}\right), \ell=1,2,3, & \beta_{\ell}^{j k} \sim \mathrm{N}\left(\beta_{\ell}^{k},\left(\sigma_{\beta_{\ell}}^{k}\right)^{2}\right), \ell=1,2 .
\end{array}
$$

This additional structure allows us to quantify differences between treatments for parameters of interest by looking at posterior distributions of the parameters in this second layer of the hierarchy.

\subsection{Inference}

Before proceeding with a Bayesian analysis, we need to specify a prior distribution for the parameters governing the model for the observable quantities in (7) and (8) and the random effects distributions (9). We adopt the following independent semi-conjugate prior distributions for each parameter: for $k=1,2$

$$
\begin{aligned}
\mu^{k} & \sim \mathrm{N}\left(b_{\mu}, d_{\mu}^{2}\right), & \left(\sigma_{\mu}^{k}\right)^{-2} & \sim \Gamma\left(g_{\mu}, h_{\mu}\right), \\
\theta_{\ell}^{k} & \sim \mathrm{N}\left(b_{\theta_{\ell}}, d_{\theta_{\ell}}^{2}\right), & \left(\sigma_{\theta_{\ell}}^{k}\right)^{-2} & \sim \Gamma\left(g_{\theta_{\ell}}, h_{\theta_{\ell}}\right), \ell=1,2 \\
\delta_{\ell}^{k} & \sim \mathrm{N}\left(b_{\delta_{\ell}}, d_{\delta_{\ell}}^{2}\right), & \left(\sigma_{\delta_{\ell}}^{k}\right)^{-2} & \sim \Gamma\left(g_{\delta_{\ell}}, h_{\delta_{\ell}}\right), \ell=1,2,3 \\
\beta_{\ell}^{k} & \sim \mathrm{N}\left(b_{\beta_{\ell}}, d_{\beta_{\ell}}^{2}\right), & \left(\sigma_{\beta_{\ell}}^{k}\right)^{-2} & \sim \Gamma\left(g_{\beta_{\ell}}, h_{\beta_{\ell}}\right), \ell=1,2 \\
\left(\sigma_{y}^{k}\right)^{-2} & \sim \Gamma\left(g_{y}, h_{y}\right), & \left(\sigma_{x}^{k}\right)^{-2} & \sim \Gamma\left(g_{x}, h_{x}\right) .
\end{aligned}
$$

This choice of prior distribution allows us to fit the model using a Gibbs sampler; see Appendix B for details of the parameter full conditional distributions.

As before, values for these prior distribution parameters were solicited from our experimental colleagues (KMC and TvZ). We concluded that we had weak prior beliefs about the parameters associated with the random effect distributions for $\beta_{2}, \theta_{1}$ and $\theta_{2}$ and represent these by taking $b_{\beta_{2}}=b_{\theta_{1}}=b_{\theta_{2}}=0, d_{\beta_{2}}=d_{\theta_{1}}=d_{\theta_{2}}=30, g_{\beta_{2}}=g_{\theta_{1}}=g_{\theta_{2}}=10$ and $h_{\beta_{2}}=h_{\theta_{1}}=h_{\theta_{2}}=1$. We were fairly confident that the group mean basal temperature level $\beta_{1}^{k}$ is around $36^{\circ} C$ but less sure about the variability $\sigma_{\beta_{1}}^{k}$ of mice around this value, and so we take $b_{\beta_{1}}=36, d_{\beta_{1}}=1, g_{\beta_{1}}=10$ and $h_{\beta_{1}}=1$. Our beliefs about $\mu^{k}$ and $\sigma_{\mu}^{k}$ were also fairly weak and incorporated via $b_{\mu}=3, d_{\mu}=1$ and $g_{\mu}=10, h_{\mu}=1$. Beliefs about the quadratic function describing the disturbance during the disruptive period were that it would have roots around $9 \mathrm{am}$ and $1 \mathrm{pm}$ with a peak height of around 2, that is, would look like $C(t)=\left\{-0.5 d(t)^{2}+11 d(t)-58.5\right\} I(t)$. We could have chosen to describe our prior uncertainty about this function in terms of the locations of its roots and its peak value. However, there is some benefit (in the MCMC scheme) in retaining the normalinverse gamma structure for the quadratic's coefficients outlined above. That said, care was needed in specifying the $\delta_{\ell}^{k}$-parameters to ensure that, for example, low prior probability 
was assigned to a positive value for the coefficient of quadratic terms. We concluded that appropriate values were $b_{\delta_{1}}=-0.5, b_{\delta_{2}}=11, b_{\delta_{3}}=-58.5, d_{\delta_{1}}=0.1, d_{\delta_{2}}=1, d_{\delta_{3}}=10$ and $g_{\delta_{1}}=g_{\delta_{3}}=g_{\delta_{2}}=3, h_{\delta_{1}}=0.2, h_{\delta_{2}}=60$ and $h_{\delta_{3}}=1800$. Incidentally, this choice results in $\operatorname{Pr}\left(\delta_{1}^{k}>0\right) \simeq 10^{-6}$ and $\operatorname{Pr}\left(\delta_{1}^{j k}>0\right) \simeq 0.06$. Finally, the prior distributions we take for the error variances follow those used previously, with $g_{y}=g_{x}=1$ and $h_{y}=h_{x}=0.05$.

\subsection{Results}

We found that running the Gibbs sampler with $10 \mathrm{~K}$ iterations for burn-in and then a further $100 \mathrm{~K}$ iterations (without thinning) gave converged output with sufficiently low autocorrelations. Table 3 gives the posterior means and standard deviations for the parameters in the random effects distributions. Looking at the posterior means for overall activity level $\left(\mu^{k}\right)$, the mice in the AL group exhibit higher activity than in the CR group: 3.168 compared with 2.621. The AL mice also have a higher basal mean temperature level $\left(\beta_{1}^{k}\right): 34.876$ compared with 34.501. These differences are relatively large when compared with the posterior standard deviations of these parameters: $0.123,0.118,0.118$ and 0.115 respectively. Despite the difference in basal temperature, there appears to be little difference in the slope coefficient $\beta_{2}$ characterising the relationship between temperature and activity. Posterior distributions for the random effects standard deviation parameters appear similar across each group. However, there appear to be different levels in variation about the sinusoidal pattern for activity and temperature between groups $\left(\sigma_{x}\right.$ and $\left.\sigma_{y}\right)$. For example, the posterior mean for $\sigma_{x}$ is 1.112 in the AL group and 1.461 in the CR group. The corresponding figures for $\sigma_{y}$ are $0.385(\mathrm{AL})$ and 0.591 (CR). Again these differences are large when compared to their posterior standard deviations $(0.009,0.009,0.003$ and 0.005 respectively) and is consistent with a previous comment in section 3.3 that there is more variation within the data in the CR group than in the AL group.

Figure 3 provides kernel density estimates of the posterior densities of the group specific means for the biologically meaningful parameters in the random effects model, that is, the overall activity level $\mu^{k}$, amplitude $A^{k}$, phase shift $B^{k}$, basal temperature $\beta_{1}^{k}$, temperature slope $\beta_{2}^{k}$ and the noise levels $\sigma_{x}^{k}$ and $\sigma_{y}^{k}$. The figure underscores the comments made from Table 3 but also highlights the higher diurnal variation in the AL group with the posterior for mean amplitude moved to the right compared to the that obtained for the CR group. Moreover, we see a large difference in average phase shift suggesting that diurnal peak activity in CR mice was moved approximately 4.3 hours earlier than that for AL mice, indicating that $\mathrm{CR}$ mice became active earlier in the circadian cycle, perhaps anticipating the arrival of food. We now turn to the effect of the disruptive period. Using the output of the Gibbs sampler, we can determine the posterior distribution of the peak height of the quadratic function $\delta_{3}^{k}-\left(\delta_{2}^{k}\right)^{2} /\left(4 \delta_{1}^{k}\right)$ during the disruptive period. This distribution has mean of 1.348 in the AL group and 5.236 in the CR group and standard deviation of 0.059 and 0.591 respectively. This suggests, perhaps not surprisingly, that the mice in the CR group were more disturbed during the disruptive period than those in the AL group.

We examined the sensitivity of our results to modest changes in the prior specification, particularly for the parameters in the second layer of the hierarchical model as these can be overly influential even when using weakly informative or non-informative priors if the number of individuals in each group is small or the variance components are close to zero (Gelman, 2006). We considered a range of scenarios. For the group mean parameters $\theta_{\ell}$ and $\beta_{\ell}$, the informative priors were made more vague and the vague priors were made more informative by taking values for the $d$. between 1 and 30. The priors for the group variance 
Table 3. Posterior means and standard deviations for the parameters in the random effects distributions.

\begin{tabular}{|c|rc|rc|}
\hline & \multicolumn{2}{|c|}{$k=1$ (AL group) } & \multicolumn{2}{c|}{$k=2$ (CR group) } \\
Parameter & Mean & $(\mathrm{SD})$ & Mean & $(\mathrm{SD})$ \\
\hline$\mu^{k}$ & 3.168 & $(0.125)$ & 2.621 & $(0.118)$ \\
$\sigma_{\mu}^{k}$ & 0.327 & $(0.048)$ & 0.307 & $(0.044)$ \\
$\theta_{1}^{k}$ & -0.161 & $(0.122)$ & 0.773 & $(0.128)$ \\
$\sigma_{\theta_{1}}^{k}$ & 0.324 & $(0.048)$ & 0.329 & $(0.048)$ \\
$\theta_{2}^{k}$ & -1.219 & $(0.118)$ & -0.424 & $(0.118)$ \\
$\sigma_{\theta_{2}}^{k}$ & 0.302 & $(0.045)$ & 0.318 & $(0.048)$ \\
$\delta_{1}^{k}$ & -0.354 & $(0.067)$ & -0.436 & $(0.068)$ \\
$\sigma_{\delta}^{k}$ & 0.214 & $(0.051)$ & 0.239 & $(0.053)$ \\
$\delta_{2}^{k}$ & 9.596 & $(0.977)$ & 10.234 & $(0.902)$ \\
$\sigma_{\delta_{2}}^{k}$ & 4.782 & $(1.185)$ & 4.911 & $(1.114)$ \\
$\delta_{3}^{k}$ & -40.166 & $(7.222)$ & -47.575 & $(7.677)$ \\
$\sigma_{\delta_{3}}^{k}$ & 22.360 & $(5.524)$ & 25.746 & $(6.527)$ \\
$\beta_{1}^{k}$ & 34.876 & $(0.118)$ & 34.501 & $(0.115)$ \\
$\sigma_{\beta_{1}}^{k}$ & 0.303 & $(0.045)$ & 0.300 & $(0.043)$ \\
$\beta_{2}^{k}$ & 0.335 & $(0.109)$ & 0.366 & $(0.108)$ \\
$\sigma_{\beta_{2}}^{k}$ & 0.286 & $(0.041)$ & 0.286 & $(0.041)$ \\
$\sigma_{x}^{k}$ & 1.112 & $(0.009)$ & 1.461 & $(0.009)$ \\
$\sigma_{y}^{k}$ & 0.385 & $(0.003)$ & 0.591 & $(0.005)$ \\
\hline
\end{tabular}

components were varied by first making them more vague about the same mean by, for example, taking $g .=5$ and $h .=0.5$ and then by changing the mean to be 1 but making them increasingly vague by, for example, taking the $(g ., h)=.(0.1,0.1)$ or $(0.001,0.001)$. We found that inferences for the group mean and variance parameters were fairly insensitive to these choices, with those for the group means being particularly robust against changes in the priors for the variance components.

Finally, we assess model fit by comparing the data at each time point with their corresponding (marginal) predictive distributions for each level in the random effects model. We obtain the predictive distribution for the activity level $\left(X_{t}^{\cdot k}\right)$ for group $k$ by taking the model (8) and integrating over the random effects distribution (9) and the posterior uncertainty for their unknown (group specific) parameters. Realisations from this predictive distribution can be obtained by repeatedly using draws from the posterior sample to simulate random effect parameter values and then to simulate a trace of the activity level. The predictive distribution for the temperature level $\left(Y_{t}^{\cdot k}\right)$ for group $k$ can be determined in a similar manner. Realisations from the predictive distributions for activity, $X_{t}^{j k}$, and temperature given activity, $Y_{t}^{j k} \mid X_{t}^{j k}$, for a particular mouse $j$ in group $k$ can be obtained by averaging simulated traces of $X_{t}^{j k}$ and $Y_{t}^{j k} \mid X_{t}^{j k}$ over posterior draws of the parameters in (7) and (8).

Figure 4 shows the marginal predictive mean and 95\% credible intervals for activity and temperature levels for each group in the top two panels and those for the first mouse in each group in the bottom two panels. The first 96 data points measured on the first mouse in each group are also overlayed for comparison, with the disruptive periods removed for clarity. The model appears to fit the data reasonably well, with nearly all observations falling with the $95 \%$ credible intervals in each case. Naturally, we see more variability in the group level predictive distributions than those at the individual mouse level. The data 

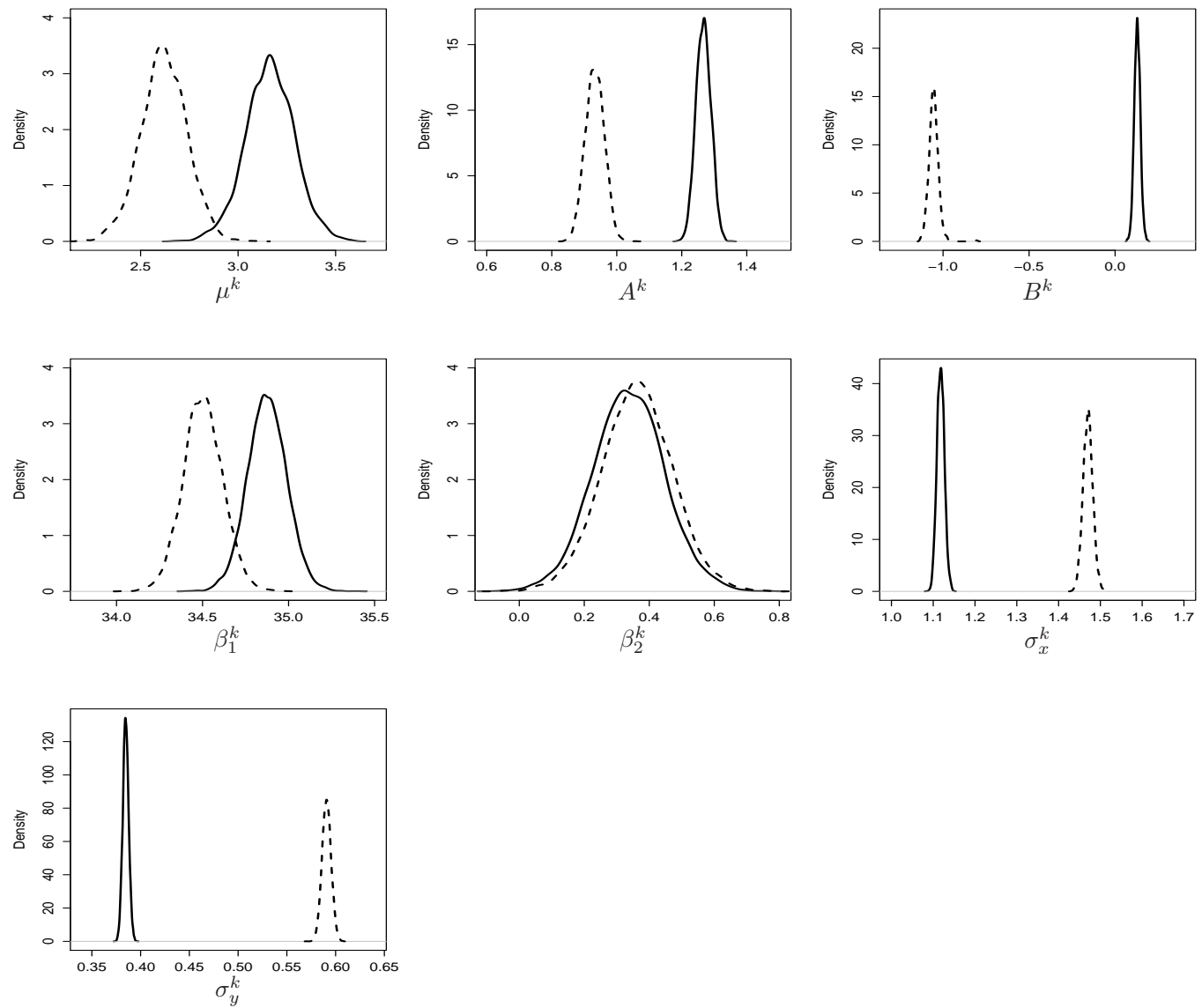

Fig. 3. Posterior densities of the group specific means for the overall activity level $\mu^{k}$, amplitude $A^{k}$, phase shift $B^{k}$, basal temperature $\beta_{1}^{k}$, temperature slope $\beta_{2}^{k}$ and the noise levels $\sigma_{x}^{k}$ and $\sigma_{y}^{k}$ in the random effects model. Solid lines: $k=1$ (AL group); dotted lines: $k=2$ (CR group).

traces for the remaining mice all show similar levels of fit to the predictive distributions.

\section{Discussion}

We have developed a joint model for physical activity and core body temperature based on data arising from a 70 day study into the effect of late onset short term caloric restriction on mice. Our model captures the observed linear relationship between temperature and (transformed) activity and also the sinusoidal pattern due to the circadian cycle in both the activity and temperature data. After assessing temporal dependence of the model parameters, a hierarchical approach was used to quantify differences between mice fed ad libitum and those that were caloric restricted. Predictive distributions suggest that our model provides an adequate description of the data. 
(a) AL group
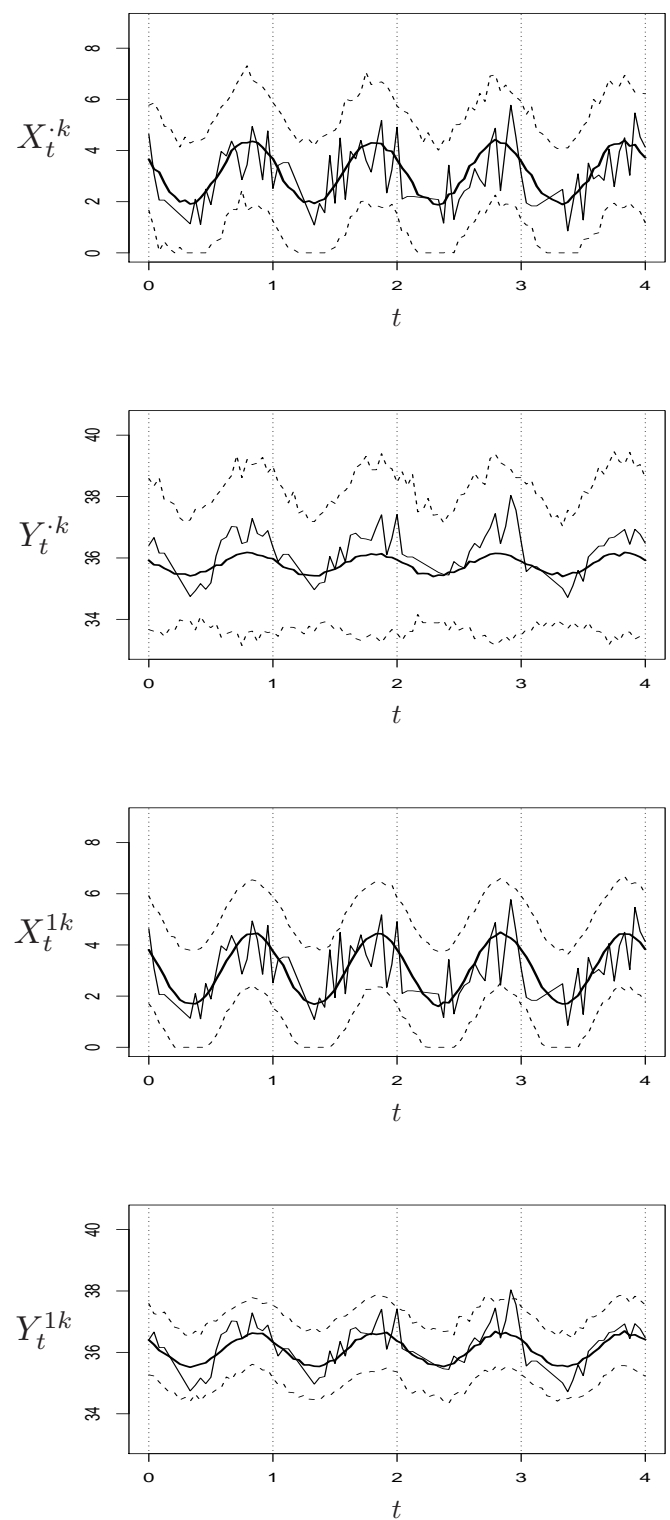

(b) CR group
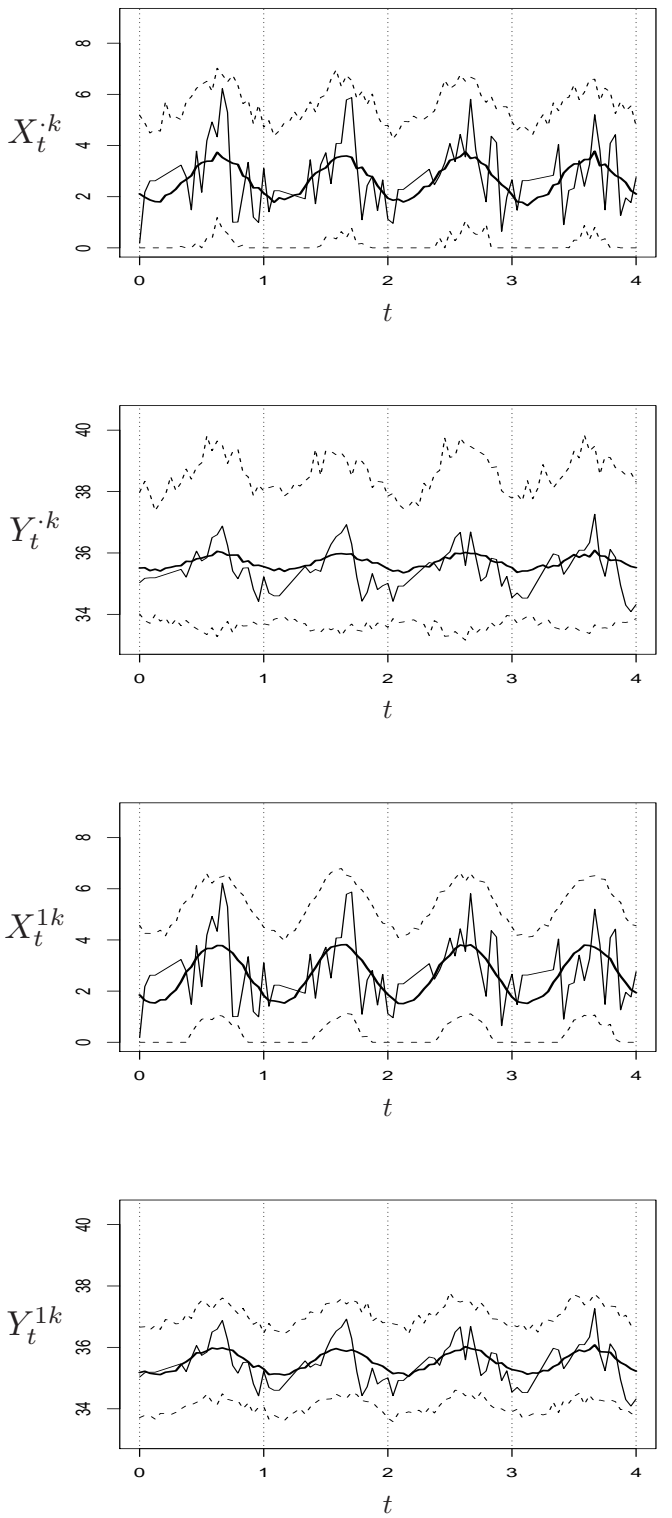

Fig. 4. Top two panels: Group level marginal predictive mean (thick solid line) and $95 \%$ credible intervals (dashed lines) for activity $X_{t}^{\cdot k}$ and temperature $Y_{t}^{\cdot k}$. Bottom two panels: Mouse 1 marginal predictive mean (thin solid line) and $95 \%$ credible intervals (dashed lines) for activity $X_{t}^{1 k}$ and temperature $Y_{t}^{1 k}$. Panels (a) and (b) correspond to the $\mathrm{AL}(k=1)$ and $\mathrm{CR}(k=2)$ groups, with data on mouse 1 in each group overlayed (thin solid line). Data during the disruptive periods have been removed. 
Although it has been reported in the literature that rodents may increase physical activity during CR due to food searching behaviour (Duffy et al., 1997; McCarter et al., 1997), we found that overall activity decreased in CR mice, and this agrees with the findings in some previous rodent (Hambly and Speakman, 2005; Severinsen and Munch, 1999) and monkey (Kemnitz et al., 1993) studies. In addition, we found that diurnal variation was higher in AL mice but that the diurnal rhythm of CR mice was significantly earlier than in AL mice, possibly due to a stronger response to the period of food availability.

Basal core body temperature generally showed a decrease during caloric restriction, as found in other related work (Weindruch and Walford, 1988; Duffy et al., 1997; Roth et al., 2002). However, our findings suggest that a reduction in core temperature is not only due to lower physical activity. Rather, basal core body temperature appeared to be fixed at a lower set-point in CR mice despite there being little difference in the linear coefficient characterising the relationship between temperature and (transformed) activity between $\mathrm{AL}$ and $\mathrm{CR}$ mice. Finally we found that $\mathrm{CR}$ mice were more disturbed during the morning disruptive period than those in the AL group. This is consistent with Duffy et al. (1989) who found that $\mathrm{CR}$ rats had a substantial increase in body temperature at feeding time.

The results presented in this paper are those from a pilot study. We look forward to seeing data from a longer study involving many more mice. It will be interesting to see if these larger data sets confirm our findings and also allow analysts to detect other aspects such as gender differences and differing reactions during the dark and light phases of the day. Examining the effects of long term and early onset caloric restriction will also of interest.

\section{Acknowledgements}

We thank the Joint Editor, the Associate Editor and three referees for their thoughtful and constructive comments which greatly improved the paper. The authors are affiliated with the Centre for Integrated Systems Biology of Ageing and Nutrition (CISBAN) at Newcastle University, which is supported jointly by the Biotechnology and Biological Sciences Research Council (BBSRC) and the Engineering and Physical Sciences Research Council (EPSRC).

\section{A. MCMC scheme for the non-centered parameterisation}

We consider the task of sampling jointly the indicator $\gamma$, the parameters in the full (unrestricted) model $\boldsymbol{\psi}$ and the time varying parameters $\tilde{\boldsymbol{\beta}}$ and $\tilde{\boldsymbol{\theta}}$ in the non-centered parameterisation given by (5) and (6).

Conditional on the latent process $\tilde{\boldsymbol{\beta}}$, the observation equation for the conditional temperature model in (5) can be written as a standard regression

$$
Y_{t_{i}}=\boldsymbol{Z}_{t_{i}}^{\gamma, y} \boldsymbol{\psi}_{y}^{\gamma}+\sigma_{y} \epsilon_{i}^{y}, \quad \epsilon_{i}^{y} \sim \mathrm{N}(0,1)
$$

where, if $\gamma=1, \boldsymbol{Z}_{t_{i}}^{\gamma, y}=\left(1, x_{t_{i}}, \tilde{\beta}_{t_{i}, 1}+\tilde{\beta}_{t_{i}, 2} x_{t_{i}}\right), \boldsymbol{\psi}_{y}^{\gamma}=\left(\beta_{1}, \beta_{2}, \tilde{\sigma}_{\beta, 1}, \tilde{\sigma}_{\beta, 2}\right)^{\prime}$, and if $\gamma=0$, $\boldsymbol{Z}_{t_{i}}^{\gamma, y}=\left(1, x_{t_{i}}\right)$ and $\boldsymbol{\psi}_{y}^{\gamma}=\left(\beta_{1}, \beta_{2}\right)^{\prime}$. Similarly, conditional on the latent process $\tilde{\boldsymbol{\theta}}$, the observation equation for the marginal (transformed) activity model in (5) can be written as

$$
X_{t_{i}}=\boldsymbol{Z}_{t_{i}}^{\gamma, x} \boldsymbol{\psi}_{x}^{\gamma}+\sigma_{x} \epsilon_{i}^{x}, \quad \epsilon_{i}^{x} \sim \mathrm{N}(0,1)
$$

where, if $\gamma=1, \boldsymbol{Z}_{t_{i}}^{\gamma, x}=\left(1, \sin \left(\pi t_{i} / 12\right), \cos \left(\pi t_{i} / 12\right), \tilde{\theta}_{t_{i}, 1} \sin \left(\pi t_{i} / 12\right)+\tilde{\theta}_{t_{i}, 2} \cos \left(\pi t_{i} / 12\right)\right)$, $\boldsymbol{\psi}_{x}^{\gamma}=\left(\mu_{x}, \theta_{1}, \theta_{2}, \tilde{\sigma}_{\theta, 1}, \tilde{\sigma}_{\theta, 2}\right)^{\prime}$, and if $\gamma=0, \boldsymbol{Z}_{t_{i}}^{\gamma, x}=\left(1, \sin \left(\pi t_{i} / 12\right), \cos \left(\pi t_{i} / 12\right)\right)$ and $\boldsymbol{\psi}_{x}^{\gamma}=$ 
$\left(\mu_{x}, \theta_{1}, \theta_{2}\right)^{\prime}$. Hence, conditional on $\gamma$, the latent time varying parameters and the data, $\boldsymbol{\psi}_{y}^{\gamma}$, $\sigma_{y}$, and $\boldsymbol{\psi}_{x}^{\gamma}, \sigma_{x}$ can readily be sampled from standard distributions using, for example, the results in Gamerman and Lopes (2006).

To sample the indicator $\gamma$, we follow Frühwirth-Schnatter and Wagner (2010) and marginalise over the parameters for which variable selection is performed. For the conjugate prior with $\boldsymbol{\psi}_{j}^{\gamma} \sim \mathrm{N}\left(\boldsymbol{b}_{0}^{\gamma, j}, \boldsymbol{D}_{0}^{\gamma, j} \sigma_{j}^{2}\right), \sigma_{j}^{-2} \sim \Gamma\left(g_{j}, h_{j}\right), j \in\{x, y\}$, we obtain

$$
\pi(\gamma \mid \boldsymbol{x}, \boldsymbol{y}, \tilde{\boldsymbol{\beta}}, \tilde{\boldsymbol{\theta}}) \propto \frac{\left|\boldsymbol{D}^{\gamma, x}\right|^{1 / 2}}{\left|\boldsymbol{D}_{0}^{\gamma, x}\right|^{1 / 2}} \frac{\Gamma\left(G_{x}\right) h_{x}^{g_{x}}}{\Gamma\left(g_{x}\right)\left(H_{x}^{\gamma}\right)^{G_{x}}} \times \frac{\left|\boldsymbol{D}^{\gamma, y}\right|^{1 / 2}}{\left|\boldsymbol{D}_{0}^{\gamma, y}\right|^{1 / 2}} \frac{\Gamma\left(G_{y}\right) h_{y}^{g_{y}}}{\Gamma\left(g_{y}\right)\left(H_{y}^{\gamma}\right)^{G_{y}}}
$$

where

$$
\begin{array}{rlrl}
\boldsymbol{D}^{\gamma, x} & =\left\{\left(\boldsymbol{Z}^{\gamma, x}\right)^{\prime} \boldsymbol{Z}^{\gamma, x}+\left(\boldsymbol{D}_{0}^{\gamma, x}\right)^{-1}\right\}^{-1}, & \boldsymbol{D}^{\gamma, y} & =\left\{\left(\boldsymbol{Z}^{\gamma, y}\right)^{\prime} \boldsymbol{Z}^{\gamma, y}+\left(\boldsymbol{D}_{0}^{\gamma, y}\right)^{-1}\right\}^{-1}, \\
\boldsymbol{b}^{\gamma, x} & =\boldsymbol{D}^{\gamma, x}\left\{\left(\boldsymbol{Z}^{\gamma, x}\right)^{\prime} \boldsymbol{x}+\left(\boldsymbol{D}_{0}^{\gamma, x}\right)^{-1} \boldsymbol{b}_{0}^{\gamma, x}\right\}, & \boldsymbol{b}^{\gamma, y} & =\boldsymbol{D}^{\gamma, y}\left\{\left(\boldsymbol{Z}^{\gamma, y}\right)^{\prime} \boldsymbol{y}+\left(\boldsymbol{D}_{0}^{\gamma, y}\right)^{-1} \boldsymbol{b}_{0}^{\gamma, y}\right\}, \\
G_{x}= & g_{x}+n / 2, & G_{y}=g_{y}+n / 2, \\
H_{x}^{\gamma}=h_{x}+\frac{1}{2}\left\{\boldsymbol{x}^{\prime} \boldsymbol{x}+\left(\boldsymbol{b}_{0}^{\gamma, x}\right)^{\prime}\left(\boldsymbol{D}_{0}^{\gamma, x}\right)^{-1} \boldsymbol{b}_{0}^{\gamma, x}\right. & H_{y}^{\gamma}=h_{y}+\frac{1}{2}\left\{\boldsymbol{y}^{\prime} \boldsymbol{y}+\left(\boldsymbol{b}_{0}^{\gamma, y}\right)^{\prime}\left(\boldsymbol{D}_{0}^{\gamma, y}\right)^{-1} \boldsymbol{b}_{0}^{\gamma, y}\right. \\
& \left.-\left(\boldsymbol{b}^{\gamma, x}\right)^{\prime}\left(\boldsymbol{D}^{\gamma, x}\right)^{-1} \boldsymbol{b}^{\gamma, x}\right\}, & & \left.-\left(\boldsymbol{b}^{\gamma, y}\right)^{\prime}\left(\boldsymbol{D}^{\gamma, y}\right)^{-1} \boldsymbol{b}^{\gamma, y}\right\},
\end{array}
$$

where $\boldsymbol{Z}^{\gamma, x}$ and $\boldsymbol{Z}^{\gamma, y}$ are regressor matrices with rows equal to $\boldsymbol{Z}_{t_{i}}^{\gamma, x}$ and $\boldsymbol{Z}_{t_{i}}^{\gamma, y}$ respectively.

Finally, we draw the time varying states, $\tilde{\boldsymbol{\beta}}$ and $\tilde{\boldsymbol{\theta}}$ conditional on the indicator $\gamma$ and the remaining fixed parameters. If $\gamma=0$, sampling from the prior is performed. If $\gamma=1$, we use a forwards filtering, backwards sampling (FFBS) scheme (Carter and Kohn, 1994; Frühwirth-Schnatter, 1994; Shephard, 1994; West and Harrison, 1997).

Algorithmically, the scheme proceeds using the following steps.

(a) Sample the indicator $\gamma$ and the remaining fixed parameters $\boldsymbol{\psi}^{\gamma, x}, \boldsymbol{\psi}^{\gamma, y}, \sigma_{x}$ and $\sigma_{y}$ jointly in one block:

1. Sample $\gamma$ from $\pi(\gamma \mid \boldsymbol{x}, \boldsymbol{y}, \tilde{\boldsymbol{\beta}}, \tilde{\boldsymbol{\theta}})$.

2. Draw $\sigma_{x}^{-2} \sim \Gamma\left(G_{x}, H_{x}^{\gamma}\right)$ and $\sigma_{y}^{-2} \sim \Gamma\left(G_{y}, H_{y}^{\gamma}\right)$. Draw $\boldsymbol{\psi}_{x}^{\gamma} \sim \mathrm{N}\left(\boldsymbol{b}^{\gamma, x}, \boldsymbol{D}^{\gamma, x} \sigma_{x}^{2}\right)$ and $\boldsymbol{\psi}_{y}^{\gamma} \sim \mathrm{N}\left(\boldsymbol{b}^{\gamma, y}, \boldsymbol{D}^{\gamma, y} \sigma_{y}^{2}\right)$.

3. If $\gamma=0$, set $\tilde{\boldsymbol{\sigma}}_{\beta}=\tilde{\boldsymbol{\sigma}}_{\theta}=\mathbf{0}$.

(b) If $\gamma=0$, sample $\tilde{\boldsymbol{\beta}}$ and $\tilde{\boldsymbol{\theta}}$ from their respective prior distributions. If $\gamma=1$, sample $\tilde{\boldsymbol{\beta}}$ conditional on $\boldsymbol{\psi}_{y}^{\gamma}, \sigma_{y}$ and the data via a FFBS scheme. Sample $\tilde{\boldsymbol{\theta}}$ conditional on $\boldsymbol{\psi}_{x}^{\gamma}, \sigma_{x}$ and the data via a FFBS scheme.

(c) For $j=1,2$, perform a random sign switch for $\tilde{\sigma}_{\beta, j}$ and the $\tilde{\beta}_{t_{i}, j}$, that is, with probability 0.5 , replace their current values with $-\tilde{\sigma}_{\beta, j}$ and $-\tilde{\beta}_{t_{i}, j}$. Also perform another random sign change for $\tilde{\sigma}_{\theta, j}$ and the $\tilde{\theta}_{t_{i}, j}$.

Note that after running the algorithm, draws of the time varying parameters in the centered model $(\boldsymbol{\beta}$ and $\boldsymbol{\theta}$ ) can be obtained easily from draws of $\tilde{\boldsymbol{\beta}}$ and $\tilde{\boldsymbol{\theta}}$. We implemented the algorithm in $\mathrm{R}$ (version 2.10.1) using our own functions. However, we note that step (b) can be performed using the R package "dlm" (Petris and Petrone, 2011). 


\section{B. Full conditional distributions for the random effects model parameters}

For notational simplicity, the following appendix uses precision parameters rather than variance parameters, for example, $\tau_{\theta}$ rather than $\sigma_{\theta}^{2}$.

\section{B.1. Derivation of $\pi\left(\mu^{j k}, \theta_{1}^{j k}, \theta_{2}^{j k}, \delta_{1}^{j k}, \delta_{2}^{j k}, \delta_{3}^{j k} \mid \cdot\right)$}

Consider mouse $j$ in treatment group $k$. Let

$$
\begin{aligned}
\boldsymbol{x}_{j k} & =\left(x_{1}^{j k}, x_{2}^{j k}, \ldots, x_{n}^{j k}\right)^{\prime}, & & \boldsymbol{\theta}_{j k}=\left(\mu^{j k}, \theta_{1}^{j k}, \theta_{2}^{j k}, \delta_{1}^{j k}, \delta_{2}^{j k}, \delta_{3}^{j k}\right)^{\prime}, \\
\boldsymbol{b}_{0} & =\left(\mu^{k}, \theta_{1}^{k}, \theta_{2}^{k}, \delta_{1}^{k}, \delta_{2}^{k}, \delta_{3}^{k}\right)^{\prime}, & & \boldsymbol{D}_{0}=\operatorname{diag}\left(\tau_{\mu}^{k}, \tau_{\theta_{1}}^{k}, \tau_{\theta_{2}}^{k}, \tau_{\delta_{1}}^{k}, \tau_{\delta_{2}}^{k}, \tau_{\delta_{3}}^{k}\right)
\end{aligned}
$$

and so we need $\pi\left(\boldsymbol{\theta}_{j k} \mid \cdot\right)$. We can write the marginal model for square root activity as

$$
X_{i}^{j k}=\boldsymbol{F}_{i}^{\prime} \boldsymbol{\theta}_{j k}+\sigma_{x}^{k} \epsilon_{i j k}^{x},
$$

where

$$
\boldsymbol{F}_{i}=\left(1, \sin \left(\pi t_{i} / 720\right), \cos \left(\pi t_{i} / 720\right), I\left(t_{i}\right) d\left(t_{i}\right)^{2}, I\left(t_{i}\right) d\left(t_{i}\right), I\left(t_{i}\right)\right)^{\prime} .
$$

For fixed $\boldsymbol{b}_{0}, \boldsymbol{D}_{0}$ and $\tau_{x}^{k}$, this is a linear regression model with prior distribution $\boldsymbol{\theta}_{j k} \sim$ $\mathrm{N}\left(\boldsymbol{b}_{0}, \boldsymbol{D}_{0}^{-1}\right)$. Thus

$$
\boldsymbol{\theta}_{j k} \mid \cdot \sim \mathrm{N}\left(\boldsymbol{b}_{1}, \boldsymbol{D}_{1}^{-1}\right),
$$

where $\boldsymbol{b}_{1}=\boldsymbol{D}_{1}^{-1}\left(\boldsymbol{D}_{0} \boldsymbol{b}_{0}+\tau_{x}^{k} \boldsymbol{X}^{\prime} \boldsymbol{x}_{j k}\right), \boldsymbol{D}_{1}=\boldsymbol{D}_{0}+\tau_{x}^{k} \boldsymbol{X}^{\prime} \boldsymbol{X}$ and $\boldsymbol{X}=\left(\boldsymbol{F}_{1}^{\prime}, \ldots, \boldsymbol{F}_{n}^{\prime}\right)^{\prime}$.

\section{B.2. Derivation of $\pi\left(\beta_{1}^{j k}, \beta_{2}^{j k} \mid \cdot\right)$}

Consider mouse $j$ in treatment group $k$. Let

$$
\boldsymbol{y}_{j k}=\left(y_{1}^{j k}, y_{2}^{j k}, \ldots, y_{n}^{j k}\right)^{\prime}, \quad \boldsymbol{\beta}_{j k}=\left(\beta_{1}^{j k}, \beta_{2}^{j k}\right)^{\prime}, \quad \boldsymbol{b}_{0}^{*}=\left(\beta_{1}^{k}, \beta_{2}^{k}\right)^{\prime}, \quad \boldsymbol{D}_{0}^{*}=\operatorname{diag}\left(\tau_{\beta_{1}}^{k}, \tau_{\beta_{2}}^{k}\right) .
$$

The model for temperature can be written as

$$
Y_{i}^{j k}=\boldsymbol{G}_{i}^{\prime} \boldsymbol{\beta}_{j k}+\sigma_{y}^{k} \epsilon_{i j k}^{y},
$$

where $\boldsymbol{G}_{i}=\left(1, x_{i}^{j k}\right)^{\prime}$ and we have supressed the dependence of $\boldsymbol{G}_{i}$ on $j$ and $k$. For fixed $\boldsymbol{b}_{0}^{*}$, $\boldsymbol{D}_{0}^{*}$ and $\tau_{y}^{k}$, this is a linear regression model with prior distribution $\boldsymbol{\beta}_{j k} \sim \mathrm{N}\left(\boldsymbol{b}_{0}^{*},\left[\boldsymbol{D}_{0}^{*}\right]^{-1}\right)$. Consequently

$$
\boldsymbol{\beta}_{j k} \mid \cdot \sim \mathrm{N}\left(\boldsymbol{b}_{1}^{*},\left[\boldsymbol{D}_{1}^{*}\right]^{-1}\right)
$$

where $\boldsymbol{b}_{1}^{*}=\left[\boldsymbol{D}_{1}^{*}\right]^{-1}\left(\boldsymbol{D}_{0}^{*} \boldsymbol{b}_{0}^{*}+\tau_{y}^{k} \boldsymbol{X}^{\prime} \boldsymbol{y}_{j k}\right), \boldsymbol{D}_{1}^{*}=\boldsymbol{D}_{0}^{*}+\tau_{y}^{k} \boldsymbol{X}^{\prime} \boldsymbol{X}$ and $\boldsymbol{X}=\left(\boldsymbol{G}_{1}^{\prime}, \ldots, \boldsymbol{G}_{n}^{\prime}\right)^{\prime}$.

\section{B.3. Derivation of $\pi\left(\tau_{x}^{k} \mid \cdot\right)$ and $\pi\left(\tau_{y}^{k} \mid \cdot\right)$}

Consider treatment group $k$. If $r_{i}^{j k}=x_{i}-\boldsymbol{F}_{i}^{\prime} \boldsymbol{\theta}_{j k}, i=1,2, \ldots n, j=1,2, \ldots, m$ then the $r_{i}^{j k} \mid \tau_{x}^{k}, \cdot \sim \mathrm{N}\left(0,\left[\tau_{x}^{k}\right]^{-1}\right)$ are independent. Using the prior $\tau_{x}^{k} \sim \Gamma\left(g_{x}, h_{x}\right)$ we obtain

$$
\tau_{x}^{k} \mid \cdot \sim \Gamma\left(g_{x}+\frac{m n}{2}, h_{x}+\frac{1}{2} \sum_{j=1}^{m} \sum_{i=1}^{n}\left(r_{i}^{j k}\right)^{2}\right) .
$$


Similarly

$$
\tau_{y}^{k} \mid \cdot \sim \Gamma\left(g_{y}+\frac{m n}{2}, h_{y}+\frac{1}{2} \sum_{j=1}^{m} \sum_{i=1}^{n}\left(y_{i}-\boldsymbol{G}_{i}^{\prime} \boldsymbol{\beta}_{j k}\right)^{2}\right)
$$

\section{B.4. Derivation of $\pi\left(\mu^{k} \mid \cdot\right)$ and $\pi\left(\tau_{\mu}^{k} \mid \cdot\right)$}

Consider treatment group $k$. Using the priors $\mu^{k} \sim \mathrm{N}\left(b_{\mu}, \tau_{\mu}^{-1}\right)$ and $\tau_{\mu}^{k} \sim \Gamma\left(g_{\mu}, h_{\mu}\right)$ we have

$\mu^{k} \mid \cdot \sim \mathrm{N}\left(\frac{b_{\mu} \tau_{\mu}+m \tau_{\mu}^{k} \bar{\mu}^{k}}{m \tau_{\mu}^{k}+\tau_{\mu}}, \frac{1}{m \tau_{\mu}^{k}+\tau_{\mu}}\right) \quad$ and $\quad \tau_{\mu}^{k} \mid \cdot \sim \Gamma\left(g_{\mu}+\frac{m}{2}, h_{\mu}+\frac{1}{2} \sum_{j=1}^{m}\left(\mu^{j k}-\mu^{k}\right)^{2}\right)$,

where $\bar{\mu}^{k}=\sum_{j=1}^{m} \mu^{j k} / m$.

\section{B.5. The remaining parameters}

The full conditional distributions for the remaining parameters $\theta_{1}^{k}, \theta_{2}^{k}, \delta_{1}^{k}, \delta_{2}^{k}, \delta_{3}^{k}, \beta_{1}^{k}, \beta_{2}^{k}$ and $\tau_{\theta_{1}}^{k}, \tau_{\theta_{2}}^{k}, \tau_{\delta_{1}}^{k}, \tau_{\delta_{2}}^{k}, \tau_{\delta_{3}}^{k}, \tau_{\beta_{1}}^{k}, \tau_{\beta_{2}}^{k}$ can be determined in a similar manner to that used in the above subsections.

\section{References}

Carter, C. K. and R. Kohn (1994). On Gibbs sampling for state space models. Biometrika 81, 541-553.

Challet, E., P. Pévet, N. Lakhdar-Ghazal, and A. Malan (1997). Ventromedial nuclei of the Hypothalamus are involved in the phase advance of temperature and activity rhythms in food-restricted rats fed during daytime. Brain Research Bulletin 43, 209-218.

Conti, B., M. Sanchez-Alavez, R. Winsky-Sommerer, M. C. Morale, J. Lucero, S. Brownell, V. Fabre, S. Huiton-Resendiz, S. Henriksen, E. P. Zorrilla, L. de Lecea, and T. Bartfai (2006). Transgenic mice with a reduced core body temperature have an increased life span. Science 314, 825-828.

Dixon, D. P., A. M. Ackert, and L. A. Eckel (2003). Development of, and recovery from, activity-based anorexia in female rats. Physiology \& Behavior 80, 273-279.

Duffy, P. H., R. J. Feuers, J. E. A. Leakey, K. D. Nakamura, A. Turturro, and R. W. Hart (1989). Effects of chronic caloric restriction on physiological variables related to energy metabolism in the male Fischer 344 rat. Mechanisms of Ageing Development 48, 117-133.

Duffy, P. H., J. E. A. Leakey, J. L. Pipkin, A. Turturro, and R. W. Hart (1997). The physiologic, neurologic, and behavioral effects of caloric restriction related to aging, disease, and environmental factors. Environmental Research 73, 242-248.

Frühwirth-Schnatter, S. (1994). Data augmentation and dynamic linear models. Journal of Time Series Analysis 15, 183-202. 
Frühwirth-Schnatter, S. (1995). Bayesian model discrimination and Bayes factors for linear gaussian state space models. Journal of the Royal Statistical Society, Series B 57, 237246.

Frühwirth-Schnatter, S. and R. Tüchler (2008). Bayesian parsimonious covariance estimation for hierarchical linear mixed models. Statistics and Computing 18, 1-13.

Frühwirth-Schnatter, S. and H. Wagner (2010). Stochastic model specification search for Gaussian and partial non-Gaussian state space models. Journal of Econometrics 154, 85-100.

Gamerman, D. and H. F. Lopes (2006). Markov chain Monte Carlo: Stochastic simulation for Bayesian inference (2nd ed.). London: Chapman and Hall.

Gelman, A. (2006). Prior distributions for variance parameters in hierarchical models. Bayesian Analysis 1, 515-534.

Gelman, A., J. B. Carlin, H. S. Stern, and D. B. Rubin (2003). Bayesian Data Analysis (2nd ed.). London: Chapman and Hall.

George, E. I. and R. McCulloch (1993). Variable selction via Gibbs sampling. Journal of the American Statistical Association 88, 881-889.

George, E. I. and R. McCulloch (1997). Approaches for Bayesian variable selction. Statistica Sinica 7, 339-373.

Hambly, C. and J. R. Speakman (2005). Contribution of different mechanisms to compensation for energy restriction in the mouse. Obsesity Research 13, 1548-1557.

Kass, R. E. and A. E. Raftery (1995). Bayes factors. Journal of the American Statistical Association 90, 773-795.

Kemnitz, J. W., R. Weindruch, E. B. Roecker, K. Crawford, P. L. Kaufman, and W. B. Ershler (1993). Dietary restriction of adult male rhesus monkeys: Design, methodology, and preliminary findings from the first year of study. Journal of Gerontology 48, B17-B26.

Liao, C. Y., B. A. Rikke, T. E. Johnson, V. Diaz, and J. F. Nelson (2010). Genetic variation in the murine lifespan response to dietary restriction: from life extension to life shortening. Aging Cell 9, 92-95.

McCarter, R. J. M., I. Shimokawa, Y. Ikeno, Y. Higami, G. B. Hubbard, B. P. Yu, and C. A. McMahan (1997). Physical activity as a factor in the action of dietary restriction on aging: Effects in Fischer 344 rats. Aging (Milano) 9, 73-79.

Mousel, M. R., W. W. Stroup, and M. K. Nielsen (2000). Locomotor activity, core body temperature, and circardian rhythms in mice selected for high or low heat loss. Journal of Animal Science 79, 861-868.

Petris, G. and S. Petrone (2011). State space models in R. Journal of Statistical Software 41.

Pugh, T. D., T. D. Oberley, and R. Weindruch (1999). Dietary intervention at middle age: Caloric restriction but not dehydroepianrosterone sulfate increases lifespan and lifetime cancer incidence in mice. Cancer Research 59, 1642-1648. 
Roth, G. S., M. A. Lane, D. K. Ingram, J. A. Mattison, D. Elahi, J. D. Tobin, D. Muller, and E. J. Metter (2002). Biomarkers of caloric restriction may predict longevity in humans. Science 297, 811.

Severinsen, T. and I. C. Munch (1999). Body core temperature during food restriction in rats. Acta Physiologica Scandinavica 165, 299-305.

Shephard, N. (1994). Partial non-Gaussian state space models. Biometrika 81, 115-131.

Spindler, S. R. (2005). Rapid and reversible induction of the longevity, anticancer and genomic effects of caloric restriction. Mechanisms of Ageing and Development 126, 960 966.

Volk, M. J., T. D. Pugh, M. Kim, C. H. Frith, R. A. Daynes, W. B. Ershler, and R. Weindruch (1994). Dietary restriction from middle age attenuates age-associated lymphoma development and interleukin 6 dysregulation in c57bl/6 mice. Cancer Research 54, 30543061.

Weindruch, R. H. and R. L. Walford (1982). Dietary restriction in mice beginning at 1 year of age: Effect on life-span and spontaneous cancer incidence. Science 215, 1415-1418.

Weindruch, R. H. and R. L. Walford (1988). The retardation of aging and disease by calorie-restiction. Springfield, IL, USA: Charles C. Thomas.

West, M. and P. J. Harrison (1997). Bayesian Forecasting and Dynamic Models (2nd ed.). New York: Springer Verlag.

Yu, B. P., E. J. Masoro, and C. A. McMahan (1985). Nutritional influences on aging of Fischer 344 rats: I. physical, metabolic, and longevity characteristics. Journal of Gerontology 40, 657-670. 